\title{
Studies on the Toxicity and Efficacy of a New Amino Acid Solution in Pediatric Parenteral Nutrition
}

\author{
Arnold G. Coran, M.D.,* And Robert A. Drongowski, M.S. $\dagger$ \\ From the Section of Pediatric Surgery, Mott Children's Hospital, Ann Arbor, and University of Michigan Medical School, Ann Arbor, Michigan
}

\begin{abstract}
The optimum composition and concentration of crystalline amino acid solutions necessary for growth and brain maturation in critically ill infants requiring total parenteral nutrition (TPN) are unknown. Either an excess or a deficiency of amino acids could theoretically impair normal brain development in the neonate. The purpose of this study was to compare the toxicity and efficacy of two intravenous amino acid solutions, Neopham, modeled after the amino acid pattern found in human breast milk, and Aminosyn, a marketed product, designed for general usage.

Sixteen infants and children requiring continuous intravenous nutrition for at least 7 days received the Neopham amino acid solution, and eight infants and children received the Aminosyn amino acid solution as part of a total parenteral nutrition
\end{abstract}

regimen which included glucose, the fat emulsion Intralipid, as well as routine mineral and vitamin additives.

There were no significant differences in mean gestational age, body weight, postnatal age, or mean daily nutrient intake between the patients receiving Aminosyn or Neopham. The daily nitrogen intake, excretion, and retention were similar in both groups. In addition, there were no statistically significant differences in either hematological or biochemical parameters between the two study groups.

The plasma levels of three essential amino acids, isoleucine, methionine, and valine, rose significantly higher in the Aminosyn-treated patients. The plasma levels of all the essential amino acids increased in both study groups. (Journal of Parenteral and Enteral Nutrition 11:368-377, 1987)
Glycine was the only nonessential amino acid whose plasma level was significantly higher in the Aminosyntreated children. The levels of the remaining nonessential amino acids either increased or decreased, depending upon the amino acid solution infused. In general, the plasma aminogram analyses reflected the composition of the respective parent solution.

On the basis of this study, Neopham appears to be as effective as Aminosyn in a pediatric TPN regimen in terms of weight gain, nitrogen balance, and clinical condition. No serious side effects were observed with either amino acid solution. Finally, plasma aminograms appear to reflect the amino acid composition of the infused solution.

Total parenteral nutrition (TPN) administered via a central or peripheral vein has gained wide acceptance for use in selected pediatric and adult patients. ${ }^{1}$ A typical TPN regimen includes glucose, protein (supplied in the form of crystalline amino acids), a fat emulsion, vitamins, minerals, and trace elements. Such combinations of nutrients have proven very effective in totally supplying critically ill infants with the nutritional support necessary for growth, when enteral feedings are not feasible. ${ }^{2-5}$ However, definitive studies delineating the optimum composition and concent ration of crystalline amino acid solutions necessary for growth ${ }^{6-9}$ and brain

Received for publication, June 16, 1986.

Accepted for publication. December 1, 1986

Reprint requests: Arnold G. Coran, M.D., Room F7516, Box 0245, Mott Children's Hospital. Ann Arbor, MI 48109.

- Professor of Surgery and Head of Section of Pediatric Surgery, University of Michigan Medical School; Surgeon-in-Chief, Mott Children's Hospital.

+ Senior Research Associate, Pediat ric Surgery Research Laboratories, Mott Children's Hospital, Ann Arbor. Michigan. maturation ${ }^{10-13}$ have not been performed. Amino acid deficiency or excess in the developing neonate could possibly impair normal development of the brain. ${ }^{14-16}$

The purpose of this study was to compare the toxicity and efficacy of Neopham (KabiVitrum, Inc.) with Aminosyn (Abbott Laboratories) as a nitrogen source in a TPN protocol in infants and children. The results document that Neopham is as effective as aminosyn in

TABLE I

Composition of Neopham and Aminosyn amino acid solutions

\begin{tabular}{|c|c|c|c|c|}
\hline \multirow{2}{*}{ Amino acid } & \multicolumn{2}{|c|}{ Neopham } & \multicolumn{2}{|c|}{ Aminosyn } \\
\hline & $\overline{\mathrm{g} / \mathrm{dl}}$ & \% Total & $\mathrm{g} / \mathrm{dl}$ & $\%$ Total \\
\hline l-Isoleucine & 0.31 & 4.8 & 0.51 & 7.3 \\
\hline$l$-Leucine & 0.70 & 10.8 & 0.66 & 9.5 \\
\hline$l$-Lysine & 0.56 & 8.6 & 0.51 & 7.3 \\
\hline$l$-Methionine & 0.13 & 2.0 & 0.28 & 4.0 \\
\hline$l$-Phenylalanine & 0.27 & 4.2 & 0.31 & 4.4 \\
\hline$l$-Threonine & 0.36 & 5.5 & 0.37 & 5.3 \\
\hline l-Tryptophan & 0.14 & 2.2 & 0.12 & 1.7 \\
\hline$l$-Valine & 0.36 & 5.5 & 0.56 & 8.0 \\
\hline TOTAL ESSENTIAL AMINO ACIDS & 2.83 & 43.5 & 3.22 & 46.2 \\
\hline$l$-Histidine & 0.21 & 3.2 & 0.21 & 3.0 \\
\hline l-Arginine & 0.41 & 6.3 & 0.69 & 9.9 \\
\hline l-Alanine & 0.63 & 9.7 & 0.90 & $12.9^{a}$ \\
\hline l-Proline & 0.56 & 8.6 & 0.61 & 8.8 \\
\hline l-Serine & 0.38 & 5.8 & 0.30 & 4.3 \\
\hline l-Tyrosine & 0.05 & 0.8 & 0.04 & 0.6 \\
\hline l-Glycine & 0.21 & 3.2 & 0.90 & $12.6^{a}$ \\
\hline l-Aspartic acid & 0.41 & $6.3^{a}$ & & \\
\hline l-Glutamic acid & 0.71 & $10.9^{\alpha}$ & & \\
\hline l-Cysteine-cystine & 0.10 & $1.5^{a}$ & & \\
\hline $\begin{array}{l}\text { TOTAL NONESSENTIAL AMINO } \\
\text { ACIDS }\end{array}$ & 3.67 & 56.5 & 3.75 & 53.8 \\
\hline TOTAL AMINO ACIDS & 6.50 & 100.0 & 6.97 & 100.0 \\
\hline
\end{tabular}

${ }^{-}$Denotes significant difference. 
promoting growth, as measured by weight gain and positive nitrogen balance, and that no adverse side effects of toxicity can be attributed to Neopham.

\section{MATERIALS AND METHODS}

Twenty-four infants and children, from one day to 10 yr old, who required continuous TPN for at least 7 days were studied. Patients receiving any enteral nutrition, those with a diagnosis of cardiac failure, renal insufficiency, diabetes mellitus, untreated infections, and those receiving concurrent corticosteroid medication were excluded from the study.

After obtaining parental informed consent. the following preinfusion tests were performed: urine-urea-nitrogen and urine osmolality, plasma amminogram analysis, complete blood count with differential white blood cell count, platelet count. blood-urea-nitrogen (BL $\$$ ). serum uric acid. albumin. total protein. cholesterol. triglycerides. total bilirubin. alkaline phosphatase, lactic acid dehydrogenase (LDH), serum glutamic oxaloacetic transaminase (SGOT), ammonia, magnesium, and osmolality.

TABLE II

Demographic data of patients administered Aminosyn $(n=8)$ or Neopham $(n=16)$ as the amino acid source in a TPN regimen

\begin{tabular}{|c|c|c|c|c|c|c|}
\hline \multirow{2}{*}{ Sex } & \multirow{2}{*}{$\begin{array}{c}\text { Gestational age } \\
\text { (wk) }\end{array}$} & \multirow{2}{*}{$\begin{array}{l}\text { Age at study } \\
(\mathrm{D} / \mathrm{M} / \mathrm{Y})^{a}\end{array}$} & \multirow{2}{*}{$\begin{array}{l}\text { Initial wt } \\
(\mathrm{kg})\end{array}$} & \multicolumn{2}{|c|}{ Prestudy status } & \multirow{2}{*}{ Diagnosis } \\
\hline & & & & Prognosis & Nutritional & \\
\hline \multicolumn{7}{|c|}{ Aminosyn-Treated Patients (Group 1) } \\
\hline $\mathrm{F}$ & 36 & $3 \mathrm{D}$ & 2.48 & Good & Normal & Gastroschisis \\
\hline $\mathbf{F}$ & 37 & $2 \mathrm{D}$ & 2.50 & Good & Normal & Gastroschisis, meconium pneumonitis \\
\hline $\mathbf{F}$ & 40 & 3D & 2.58 & Good & Normal & Duodenal atresia \\
\hline $\mathbf{M}$ & & $1.5 \mathrm{M}$ & 3.28 & Good & Normal & Duodenal atresia, situs inversus \\
\hline $\mathbf{F}$ & 40 & $3 \mathrm{D}$ & 2.86 & Good & Normal & Intestinal malrotation-volvulus \\
\hline M & 40 & $16 \mathrm{D}$ & 3.76 & Good & Normal & Omphalocele \\
\hline $\mathbf{M}$ & & $10 \mathrm{M}$ & 7.98 & Good & Normal & Intestinal volvulus, situs inversus \\
\hline $\mathbf{M}$ & 38 & $3 \mathrm{D}$ & 2.30 & Good & Normal & Intestinal malrotation \\
\hline \multicolumn{7}{|c|}{ Neopham-Treated Patients (Group 2) } \\
\hline $\mathbf{F}$ & & $3 \mathrm{Y}$ & 10.24 & Good & Normal & Colonic aganglionosis, enterocolitis \\
\hline $\mathbf{F}$ & 33 & $1 \mathrm{D}$ & 2.00 & Good & Normal & Intestinal atresia \\
\hline $\mathbf{F}$ & 40 & $2 \mathrm{D}$ & 2.50 & Good & Normal & Gastroschisis, meconium pneumonitis \\
\hline$F$ & 38 & $2 \mathrm{D}$ & 2.56 & Good & Normal & Gast roschisis \\
\hline $\mathbf{F}$ & & $10 \mathrm{Y}$ & 12.70 & Good & Underweight & $\begin{array}{l}\text { Hiatal hernia, esuphagitis, mental re- } \\
\text { tardation }\end{array}$ \\
\hline $\mathbf{F}$ & 38 & $18 \mathrm{D}$ & 1.68 & Good & Underweight & Intestinal at resia. imperforate anus \\
\hline $\mathbf{F}$ & 38 & $2 \mathrm{D}$ & 2.66 & Good & Normal & Intestinal atresia \\
\hline M & & $10 \mathrm{Y}$ & 21.40 & Fair & Underweight & $\begin{array}{l}\text { Esophageal stricture, mental retarda- } \\
\text { tion }\end{array}$ \\
\hline $\mathrm{F}$ & & $1 \mathbf{M}$ & 2.82 & Good & Normal & Intestinal stricture \\
\hline $\mathbf{M}$ & 34 & 2D & 2.18 & Good & Normal & Gast roschisis \\
\hline M & 40 & 1D & 2.80 & Good & Normal & Gast roschisis \\
\hline $\mathbf{F}$ & & $4 \mathrm{Y}$ & 12.20 & Poor & Underweight & $\begin{array}{l}\text { Aspiration pneumonia, anoxic brain } \\
\text { damage }\end{array}$ \\
\hline $\mathbf{M}$ & 35 & $21 \mathrm{D}$ & 2.67 & Fair & Normal & $\begin{array}{l}\text { Intestinal atresia, ventricular septal } \\
\text { defect }\end{array}$ \\
\hline $\mathbf{M}$ & 39 & $7 \mathrm{D}$ & 3.44 & Good & Normal & $\begin{array}{l}\text { Gastroschisis, respiratory distress } \\
\text { syndrome }\end{array}$ \\
\hline$M$ & & $1.5 \mathrm{Y}$ & & Fair & Normal & Trauma, multiple enterostomies \\
\hline $\mathbf{M}$ & 36 & 1D & 2.50 & Good & Normal & Gastroschisis \\
\hline
\end{tabular}

${ }^{a} \mathrm{D}=$ days, $\overline{\mathrm{M}}=$ months, $\mathrm{Y}=$ years.

TABLE III

Gestational age, body weight, and postnatal age of the Aminosyn-and Neopham-treated patients

\begin{tabular}{|c|c|c|c|}
\hline & Aminosyn & Neopham & $p$ Value \\
\hline $\begin{array}{l}\text { Neonatal analysis } \\
\text { Number } \\
\text { Male:female } \\
\text { Gestational age (wk) } \\
\text { Range } \\
\text { Postnatal age (days) } \\
\text { Range } \\
\text { Body ut (kg) } \\
\text { Range }\end{array}$ & $\begin{array}{c}6 \\
2: 4 \\
38.5 \pm 1.8 \\
36-40 \\
5.0 \pm 5.0 \\
2-6 \\
2.75 \pm 0.66 \\
2.30-3.76\end{array}$ & $\begin{array}{c}10 \\
5: 5 \\
37.1 \pm 2.5 \\
33-40 \\
5.7 \pm 7.5 \\
1-21 \\
2.50 \pm 0.52 \\
1.68-3.44\end{array}$ & $\begin{array}{l}0.26 \\
0.84 \\
0.35\end{array}$ \\
\hline $\begin{array}{l}\text { Infants and children } 28 \text { days old, or more } \\
\text { Number } \\
\text { Male:female } \\
\text { Age (mo) } \\
\text { Body wt (kg) }\end{array}$ & $\begin{array}{c}2 \\
2: 0 \\
5.75 \pm 6.01 \\
5.63 \pm 3.32\end{array}$ & $\begin{array}{c}6 \\
2: 4 \\
57.16 \pm 51.2 \\
11.87 \pm 6.64\end{array}$ & \\
\hline
\end{tabular}


Patients were randomly assigned in a $1: 2$ ratio to receive TPN solutions with Aminosyn (Abbott Laboratories). designated group 1, or Neopham (KabiVitrum, Inc.l. designated group 2, either by periperhal or central venous routes. The amino acid composition of Aminosyn and Veopham is listed in Table 1. The solutions for centrally administered TPN consisted of $25 \%$ glucose and $3 . j \dot{c}$ crystalline amino acids, whereas the peripheral regimen consisted of $12.5 \%$ glucose and $2.5^{\%}$ crystalline amino acids. Intralipid was provided to a maximum of $40 \%$ of daily caloric intake. All TPN solutions contained routine vitamin and mineral additives.

Daily observations included vital signs, weight, urine tests, and a report of adverse symptoms or side effects. Estimated caloric requirements and nitrogen balance were computed daily. At weekly intervals, blood chemistries were repeated. Blood ammonia levels and plasma aminograms were analyzed twice weekly.

Nitrogen intakes were calculated for each day of TPN from the recorded infusions of amino acids. One gram of amino acids as Aminosyn provided $157.7 \mathrm{mg}$ of nitrogen, 1.0 gram of amino acids as Neopham provided $143.1 \mathrm{mg}$ of nitrogen. The amount of nitrogen excreted was measured to the nearest $1.0 \mathrm{mg}$ on each day in which urine collections were successfully obtained. Approximate nitrogen balances were determined as the differences between nitrogen intake and nitrogen excretion. Nitrogen losses from nasogastric drainage, wounds, and fecal and enterostomy output were not quantified.

The data were subjected to two separate statistical analyses due to the heterogeneity of the population studied. The first analysis involved the 16 patients who were less than 28 days old at entrance into the study protocol (Neonatal Analysis). The second evaluation (Total Pa- tient Analysis) pertained to all 24 patients; the age range of the eight additional patients spanned 1 month to 10 years. Data from the first 15 days of TPN formed the basis of this study.

\section{Demographic Data}

Demographic data (mean $\pm \mathrm{SD}$ ) of the eight patients who received Aminosyn (group 1) and the 16 patients who received Neopham (group 2) as the amino acid component of a total hyperalimentation nutritional regimen are presented in Table II.

Neonatal Analysis. There were no significant differences in mean gestational age, body weight, or postnatal age at entrance into the study $(n=16)$ between the neonates receiving either Neopham $(n=10)$ or Aminosyn $(n=6)$ (Table III). A mean gestational age of 38.5 \pm 1.8 weeks, a mean initial body weight of $2.75 \pm 0.66$ $\mathrm{kg}$, and a mean postnatal age of $5.0 \pm 5.0$ days were recorded for the Aminosyn (group 1) patients, while the respective values for the Neopham (group 2) patients was $37.1 \pm 2.5$ weeks, $2.5 \pm 0.52 \mathrm{~kg}$, and $5.7 \pm 7.5$ days.

Analysis of Older Infants and Children. There were no significant differences in mean body weight $[5.6 \pm 3.32$ $\mathrm{kg}$ (Aminosyn) us $11.87 \pm 6.64 \mathrm{~kg}$ (Neopham)] and age at entrance into the study protocol $[5.75 \pm 6.01$ months (Aminosyn) us 57.16 \pm 51.2 months (Neopham)] between the two patient groups (Table III).

\section{Nutritional Data}

The mean daily nutrient intakes of the group 1 (Aminosyn) and group 2 (Neopham) patients are presented in Table IV. There were no significant between- or within-

TABLE IV

Mean nutrient intake in patients who received Aminosyn (group 1) or Neopham (group 2)

\begin{tabular}{|c|c|c|c|c|c|}
\hline & $\begin{array}{c}\text { Dextrose } \\
\text { (kcal/kg/day) }\end{array}$ & $\begin{array}{c}\text { Intralipid } \\
\text { (kcal/kg/day) }\end{array}$ & $\begin{array}{c}\text { Amino acids } \\
\text { (kcal/kg/day) }\end{array}$ & $\begin{array}{l}\text { Energy intake } \\
\text { (kcal/kg/day) }\end{array}$ & $\begin{array}{c}\text { Amino acids } \\
(\mathrm{g} / \mathrm{kg} / \mathrm{day})\end{array}$ \\
\hline $\begin{array}{l}\text { Neonatal analysis } \\
\text { Group } 1(\mathrm{n}=6) \\
\text { Group } 2(\mathrm{n}=10)_{p \text { Value }^{a}}\end{array}$ & $\begin{array}{c}56.8 \pm 8.7 \\
57.6 \pm 8.5 \\
0.98\end{array}$ & $\begin{array}{c}24.6 \pm 9.2 \\
23.7 \pm 11.3 \\
0.80\end{array}$ & $\begin{array}{c}11.7 \pm 3.4 \\
11.3 \pm 3.3 \\
0.75\end{array}$ & $\begin{array}{c}93.1 \pm 16.5 \\
92.7 \pm 15.6 \\
0.77\end{array}$ & $\begin{array}{c}2.92 \pm 0.9 \\
2.84 \pm 0.8 \\
0.76\end{array}$ \\
\hline $\begin{array}{l}\text { Total patient analysi } \\
\text { Group } 1(\mathrm{n}=8) \\
\text { Group } 2(\mathrm{n}=16) \\
p \text { Value }\end{array}$ & $\begin{array}{c}54.1 \pm 12.5 \\
59.0 \pm 19.2 \\
0.70\end{array}$ & $\begin{array}{c}26.6 \pm 10.5 \\
21.1 \pm 12.2 \\
0.46\end{array}$ & $\begin{array}{c}11.0 \pm 3.3 \\
10.7 \pm 4.4 \\
0.63\end{array}$ & $\begin{array}{c}91.6 \pm 18.7 \\
90.9 \pm 25.6 \\
0.79\end{array}$ & $\begin{array}{c}2.75 \pm 0.8 \\
2.67 \pm 1.1 \\
0.58\end{array}$ \\
\hline
\end{tabular}

${ }^{a} p$ values calculated by repeated measures analysis of variance.

TABLE V

Mean nitrogen balance data of patients who received either Aminosyn (group 1) or Neopham (group 2)

\begin{tabular}{|c|c|c|c|c|c|}
\hline & \multicolumn{2}{|c|}{ Intake } & \multirow{2}{*}{$\begin{array}{l}\text { Excretion } \\
(\mathrm{mg} / \mathrm{kg} / \text { day })\end{array}$} & \multirow{2}{*}{$\begin{array}{c}\text { Balance } \\
(\mathrm{mg} / \mathrm{kg} / \text { day })\end{array}$} & \multirow{2}{*}{$\begin{array}{c}r \\
\text { Retention }\end{array}$} \\
\hline & $\mathrm{mg} / \mathrm{kg} / \mathrm{day}$ & $\mathrm{kcal} / \mathrm{gN}$ & & & \\
\hline $\begin{array}{l}\text { Neonatal analysis } \\
\text { Group } 1(\mathrm{n}=6) \\
\text { Group } 2(\mathrm{n}=10) \\
p \text { Value }^{\alpha}\end{array}$ & $\begin{array}{c}476 \pm 119 \\
405 \pm 120 \\
0.27\end{array}$ & $\begin{array}{c}207 \pm 51 \\
261 \pm 102 \\
0.25\end{array}$ & $\begin{array}{c}137 \pm 63 \\
120 \pm 53 \\
0.58\end{array}$ & $\begin{array}{c}340 \pm 86 \\
285 \pm 135 \\
0.39\end{array}$ & $\begin{array}{l}71.8 \pm 8.2 \\
70.4 \pm 18.2\end{array}$ \\
\hline $\begin{array}{l}\text { Total patient analys } \\
\text { Group } 1(\mathrm{n}=8) \\
\text { Group } 2(\mathrm{n}=16) \\
\text { p Value } \\
\end{array}$ & $\begin{array}{c}458 \pm 107 \\
393 \pm 103 \\
0.17\end{array}$ & $\begin{array}{c}206 \pm 44 \\
257 \pm 85 \\
0.13\end{array}$ & $\begin{array}{c}153 \pm 61 \\
128 \pm 59 \\
0.35\end{array}$ & $\begin{array}{c}306 \pm 100 \\
264 \pm 117 \\
0.39\end{array}$ & $\begin{array}{l}66.8 \pm 13.0 \\
67.2 \pm 16.8\end{array}$ \\
\hline
\end{tabular}

" $p$ values calculated by one-way analysis of variance. 
TABle VI

Mean plasma concentrations of essential amino acids ( $\mu$ mol/liter) in neonates who received either Aminosyn or Neopham $(n=16)$

\begin{tabular}{|c|c|c|c|c|c|}
\hline & D 7 & W & Wo & & \\
\hline & Day 1 & Week 1 & Week 2 & Between group & Within group \\
\hline soleucine $(50$ & & & & & \\
\hline Aminosyn & $21 \pm 8$ & $88 \pm 38$ & $99 \pm 24$ & 0.01 & 0.0001 \\
\hline Neopham & $27 \pm 12$ & $52 \pm 23$ & $62 \pm 27$ & & \\
\hline Leucine $(19-2$ & & & & & \\
\hline Aminosyn & $67 \pm 23$ & $125 \pm 46$ & $129 \pm 27$ & 0.38 & 0.04 \\
\hline Neopham & $62 \pm 15$ & $138 \pm 46$ & $150 \pm 49$ & & \\
\hline Lysine $(80-40$ & & & & & \\
\hline Aminosyn & $116 \pm 38$ & $226 \pm 91$ & $232 \pm 54$ & 0.44 & 0.06 \\
\hline Neopham & $127 \pm 43$ & $232 \pm 78$ & $285 \pm 106$ & & \\
\hline Methionine (1 & & & & & \\
\hline Aminosyn & $25 \pm 10$ & $54 \pm 24$ & $65 \pm 17$ & 0.02 & 0.0001 \\
\hline Neopham & $31 \pm 9$ & $35 \pm 11$ & $42 \pm 17$ & & \\
\hline Phenylalanin & & & & & \\
\hline Aminosyn & $64 \pm 17$ & $75 \pm 19$ & $89 \pm 32$ & 0.76 & 0.58 \\
\hline Neopham & $74 \pm 19$ & $79 \pm 19$ & $87 \pm 21$ & & \\
\hline Threonine (1 & & & & & \\
\hline Aminosyn & $100 \pm 46$ & $311 \pm 123$ & $293 \pm 87$ & 0.51 & 0.12 \\
\hline Neopham & $90 \pm 33$ & $365 \pm 220$ & $364 \pm 222$ & & \\
\hline Valine $(150-3$ & & & & & \\
\hline Aminosyn & $102 \pm 35$ & $283 \pm 110$ & $295 \pm 58$ & 0.006 & 0.0001 \\
\hline Neopham & $98 \pm 22$ & $168 \pm 55$ & $188 \pm 70$ & & \\
\hline
\end{tabular}

${ }^{a}$ Represents normal values recalculated from Ghadimi and Pecora. ${ }^{17}$

TABLE VII

Mean plasma concentrations of essential amino acids ( $\mu \mathrm{mol} /$ liter) in patients who received either Aminasyn or Neopham $(n=24)$

\begin{tabular}{|c|c|c|c|c|c|}
\hline & \multirow{2}{*}{ Day 1} & \multirow{2}{*}{ Week 1} & \multirow{2}{*}{ Week 2} & \multicolumn{2}{|c|}{ p Values } \\
\hline & & & & Between group & Within group \\
\hline \multicolumn{6}{|c|}{ Isoleucine $(50-100 \mu \mathrm{mol} / \text { liter })^{a}$} \\
\hline Aminosyn & $21 \pm 10$ & $86 \pm 33$ & $97 \pm 20$ & 0.0005 & 0.0001 \\
\hline Neopham & $26 \pm 13$ & $50 \pm 20$ & $61 \pm 25$ & & \\
\hline \multicolumn{6}{|c|}{ Leucine $(19-200 \mu \mathrm{mol} /$ liter $)$} \\
\hline Aminosyn & $67 \pm 27$ & $119 \pm 43$ & $125 \pm 24$ & 0.17 & 0.01 \\
\hline Neopham & $58 \pm 18$ & $133 \pm 39$ & $152 \pm 53$ & & \\
\hline \multicolumn{6}{|c|}{ Lysine $(80-400 \mu \mathrm{mol} / \mathrm{liter})$} \\
\hline Aminosyn & $115 \pm 51$ & $218 \pm 84$ & $299 \pm 54$ & 0.48 & 0.12 \\
\hline Neopham & $117 \pm 47$ & $220 \pm 73$ & $263 \pm 107$ & & \\
\hline \multicolumn{6}{|c|}{ Methionine (19-30 $\mu \mathrm{mol} / \mathrm{liter})$} \\
\hline Aminosyn & $25 \pm 12$ & $53 \pm 21$ & $64 \pm 17$ & 0.001 & 0.0001 \\
\hline Neopham & $25 \pm 11$ & $31 \pm 11$ & $39 \pm 16$ & & \\
\hline \multicolumn{6}{|c|}{ Phenylalanine (29-110 $\mu \mathrm{mol} / \mathrm{liter})$} \\
\hline Aminosyn & $65 \pm 21$ & $72 \pm 19$ & $84 \pm 29$ & 0.41 & 0.21 \\
\hline Neopham & $62 \pm 21$ & $81 \pm 19$ & $89 \pm 23$ & & \\
\hline \multicolumn{6}{|c|}{ Threonine $(140 \mu \mathrm{mol} /$ liter $)$} \\
\hline Aminosyn & $115 \pm 51$ & $301 \pm 112$ & $288 \pm 78$ & 0.84 & 0.66 \\
\hline Neopham & $89 \pm 46$ & $304 \pm 187$ & $323 \pm 190$ & & \\
\hline \multicolumn{6}{|c|}{ Valine $(150-340 \mu \mathrm{mol} / \mathrm{liter})$} \\
\hline Aminosyn & $99 \pm 36$ & $275 \pm 98$ & $288 \pm 53$ & 0.0002 & 0.0001 \\
\hline Neopham & $96 \pm 27$ & $163 \pm 45$ & $188 \pm 66$ & & \\
\hline
\end{tabular}

${ }^{a}$ Represents normal values recalculated from Ghadimi and Pecora. ${ }^{17}$

group differences in dextrose, Intralipid, amino acid, or total energy intake (expressed as $\mathrm{kcal} / \mathrm{kg} / \mathrm{day}$ ) between the group 1 and group 2 patients.

Neonatal Analysis. The group 1 (Aminosyn) patients received $56.8 \pm 8.7 \mathrm{kcal} / \mathrm{kg} /$ day of dextrose, $24.6 \pm 9.2$ $\mathrm{kcal} / \mathrm{kg} /$ day of Intralipid and $11.7 \pm 3.4 \mathrm{kcal} / \mathrm{kg} /$ day of amino acids in comparison with the group 2 (Neopham) patients who received $57.6 \pm 8.5 \mathrm{kcal} / \mathrm{kg} /$ day of dextrose, $23.7 \pm 11.3 \mathrm{kcal} / \mathrm{kg} /$ day of Intralipid, and $11.3 \pm 3.3$ $\mathrm{kcal} / \mathrm{kg} /$ day of amino acids. The group 1 infants received an average of $2.92 \mathrm{~g} / \mathrm{kg} /$ day of aminosyn, while the group 2 infants received $2.84 \mathrm{~g} / \mathrm{kg} /$ day of Neopham. There were no significant differences in daily energy or amino acid intakes between the Aminosvn and Neopham neonatal patient groups.

Total Patient Analysis. The mean daily intake of dextruse was $54.1 \pm 12.5 \mathrm{kcal} / \mathrm{kg} / \mathrm{day}$, of Intralipid $26.6 \pm$ $10.5 \mathrm{kcal} / \mathrm{kg} / \mathrm{day}$, and of amino acids $11.11 \pm 3.3 \mathrm{kcal} / \mathrm{kg} /$ day in the group 1 (Aminosyn) patients. The me it dail. intake in the group 2 (Neopham) patients wa: $19.2 \mathrm{kcal} / \mathrm{kg}$ 'day of dextrose. $21.1 \pm 12.2 \mathrm{kcal} / \mathrm{kg}$ (1a) (1) Intralipid, and $10.7 \pm 4.4 \mathrm{kcal} \mathrm{kg}$ day of amino acids. The mean daily intake of Aminosyn was $2.75 \pm 0.8 \mathrm{~g} /$ $\mathrm{kg} /$ day in the group 1 patients, and $2.67 \pm 1.1 \mathrm{~g} / \mathrm{kg} /$ day 
TABLE VIII

Mean plasma concentrations of nonessential amino acids ( $\mu$ mol/iter) in neonates who received either Aminosyn or Neopham ( $n=16$ )

\begin{tabular}{|c|c|c|c|c|c|}
\hline & \multirow{2}{*}{ Day 1} & \multirow{2}{*}{ Week 1} & \multirow{2}{*}{ Week 2} & \multicolumn{2}{|c|}{$p$ Values } \\
\hline & & & & Between group & Within group \\
\hline \multicolumn{6}{|c|}{ Cystine $(12-50 \mu \mathrm{mol} / \text { liter })^{a}$} \\
\hline Aminosyn & $11 \pm 9$ & $12 \pm 7$ & $5 \pm 5$ & 0.14 & 0.004 \\
\hline Neopham & $11 \pm 9$ & $16 \pm 10$ & $12 \pm 9$ & & \\
\hline \multicolumn{6}{|c|}{ Histidine (38-130 $\mu \mathrm{mol} /$ /iter) } \\
\hline Aminosyn & $86 \pm 39$ & $93 \pm 16$ & $100 \pm 16$ & 0.34 & 0.22 \\
\hline Neopham & $70 \pm 14$ & $101 \pm 27$ & $109 \pm 20$ & & \\
\hline \multicolumn{6}{|c|}{ Taurine $(0-180 \mu \mathrm{mol} /$ liter $)$} \\
\hline Aminosyn & $108 \pm 73$ & $119 \pm 71$ & $91 \pm 50$ & 0.50 & 0.25 \\
\hline \multirow{2}{*}{\multicolumn{6}{|c|}{ Tyrosine $(17-240 \mu \mathrm{mol} / \mathrm{liter})$}} \\
\hline & & & & & \\
\hline Aminosyn & $54 \pm 26$ & $27 \pm 10$ & $26 \pm 11$ & 0.38 & 0.01 \\
\hline \multirow{2}{*}{\multicolumn{6}{|c|}{ Aspartic acid $(0-32 \mu \mathrm{mol} /$ liter $)$}} \\
\hline & & & & & \\
\hline Áminosyn & $37 \pm 15$ & $48 \pm 13$ & $46 \pm 12$ & 0.24 & 0.03 \\
\hline Neopham & $32 \pm 15$ & $51 \pm 24$ & $58 \pm 22$ & & \\
\hline \multicolumn{6}{|c|}{ Glutamic Acid $(0-320 \mu \mathrm{mol} /$ liter $)$} \\
\hline Aminosyn & $181 \pm 110$ & $279 \pm 124$ & $253 \pm 69$ & 0.18 & 0.0001 \\
\hline Neopham & $325 \pm 184$ & $342 \pm 161$ & $376 \pm 177$ & & \\
\hline \multicolumn{6}{|c|}{ Glutamine (430-1300 $\mu \mathrm{mol} / \mathrm{liter})$} \\
\hline Aminosyn & $238 \pm 191$ & $300 \pm 186$ & $338 \pm 144$ & 0.61 & 0.03 \\
\hline \multirow{2}{*}{\multicolumn{6}{|c|}{ Alanine $(11-770 \mu \mathrm{mol} / \mathrm{liter})$}} \\
\hline & & & & & \\
\hline Aminosyn & $197 \pm 67$ & $360 \pm 75$ & $386 \pm 89$ & 0.52 & 0.16 \\
\hline Neopham & $253 \pm 98$ & $346 \pm 152$ & $406 \pm 126$ & & \\
\hline \multicolumn{6}{|c|}{ Arginine $(40 \mu \mathrm{mol} /$ liter $)$} \\
\hline Aminosyn & $23 \pm 29$ & $98 \pm 92$ & $145 \pm 53$ & 0.24 & 0.02 \\
\hline Neopham & $27 \pm 24$ & $70 \pm 53$ & $103 \pm 52$ & & \\
\hline \multicolumn{6}{|c|}{ Glycine $(60-350 \mu \mathrm{mol} / \mathrm{liter})$} \\
\hline Aminosyn & $322 \pm 127$ & $695 \pm 220$ & $747 \pm 131$ & 0.0002 & 0.0001 \\
\hline \multirow{2}{*}{\multicolumn{6}{|c|}{ Proline $(110-430 \mu \mathrm{mol} / \mathrm{liter})$}} \\
\hline & & & & & \\
\hline Aminosyn & $109 \pm 29$ & $262 \pm 102$ & $291 \pm 71$ & 0.29 & 0.05 \\
\hline Neopham & $156 \pm 42$ & $273 \pm 119$ & $339 \pm 145$ & & \\
\hline \multicolumn{6}{|c|}{ Serine $(43-230 \mu \mathrm{mol} /$ liter $)$} \\
\hline Aminosyn & $159 \pm 48$ & $267 \pm 69$ & $283 \pm 95$ & 0.75 & 0.69 \\
\hline Neopham & $170 \pm 65$ & $246 \pm 94$ & $267 \pm 77$ & & \\
\hline
\end{tabular}

${ }^{a}$ Represents normal values recalculated from Ghadimi and Pecora. ${ }^{17}$

in the group 2 patients. These differences were not statistically significant.

\section{Nitrogen Balance}

The mean daily nitrogen intake, excretion, balance and percent retention are presented in Table $\mathrm{V}$.

Neonatal Analysis. The mean daily nitrogen intake in the group 1 (Aminosyn) patients was $476 \pm 119 \mathrm{mg} / \mathrm{kg}$ / day, and the mean nitrogen excretion was $137 \pm 63 \mathrm{mg} /$ $\mathrm{kg} /$ day for a positive mean balance of $340 \mathrm{mg} / \mathrm{kg} /$ day. This represents a retention of $71.8 \pm 8.2 \%$. The mean daily nitrogen intake in the group 2 (Neopham) patients was $405 \pm 120 \mathrm{mg} / \mathrm{kg} /$ day, and the mean excretion was $120 \pm 53 \mathrm{mg} / \mathrm{kg} /$ day, for a positive mean nit rogen balance of $285 \pm 135 \mathrm{mg} / \mathrm{kg} /$ day, or a mean retention of $70.4 \pm$ $18.2 \%$. The differences in intake, excretion, balance, and percent retention were not significantly different.

Total Patient Analyisis. The mean daily nitrogen intake in the group 1 (Aminosyn) patients was $458 \pm 107 \mathrm{mg}$ / $\mathrm{kg} / \mathrm{day}$, and the mean nitrogen excretion was $153 \pm 61$ $\mathrm{mg} / \mathrm{kg} /$ day, resulting in a net balance of $306 \pm 100 \mathrm{mg} /$ $\mathrm{kg} /$ day. The mean retention was $66.8 \pm 13.0 \%$. Similarly, the values for the group 2 (Neopham) patients were 393 $\pm 103 \mathrm{mg} / \mathrm{kg} /$ day nitrogen intake and $128 \pm 59 \mathrm{mg} / \mathrm{kg} /$ day nitrogen excretion, representing a net balance of 246 $\pm 117 \mathrm{mg} / \mathrm{kg} /$ day, or a retention of $67.2 \pm 16.8 \%$. These differences were not statistically significant.

\section{Body Weight}

Two of eight patients in the Aminosyn group lost weight over the course of the TPN therapy, while three of 14 patients in the Neopham study group lost weight during TPN.

Neonatal Analysis. The Aminosyn patients had an initial mean body weight of $2.75 \pm 0.53 \mathrm{~kg}$ and a final mean weight of $2.94 \pm 0.41 \mathrm{~kg}$, resulting in a mean percent weight increase of $0.87 \pm 2.33 /$ day. In contrast. the Neopham patients had an initial mean body weight of $2.5 \pm 0.48 \mathrm{~kg}$, and a post-study mean weight of $2.56 \pm$ $0.45 \mathrm{~kg}$, which represents a mean percent weight increase of $0.32 \pm 3.79 /$ day. These differences between the study groups were not statistically significant.

Total Patient Analysis. The group 1 Aminosyn patients 
had an initial mean body weight of $3.47 \pm 1.89 \mathrm{~kg}$, which of $4.5 \pm 3.96 \mathrm{~kg}$. and a final mean body weight of $4.88 \pm$ increased to a final mean body weight of $3.6 \pm 1.67 \mathrm{~kg}$. $4.68 \mathrm{~kg}$. resulting in a mean percent increase of $0.47 \pm$ with a mean percent increase of $0.65 \pm 2.36$ /day. The $3.4 \% /$ day. These changes were not statistically signifiNeopham study group had an initial mean body weight cant.

TABLE IX Mean plasma concentrations of nonessential amino acids ( $\mu \mathrm{mol} / \mathrm{liter}$ ) in patients uho received either Aminosyn or Neopham $(n=24)$

\begin{tabular}{|c|c|c|c|c|c|}
\hline & \multirow{2}{*}{ Day 1} & \multirow{2}{*}{ Week 1} & \multirow{2}{*}{ Week 2} & \multicolumn{2}{|c|}{$p$ Values } \\
\hline & & & & Between group & Within group \\
\hline \multicolumn{6}{|c|}{ Cystine $(12-50 \mu \mathrm{mol} / \mathrm{liter})^{a}$} \\
\hline Aminosyn & $12 \pm 8$ & $10 \pm 7$ & $4 \pm 5$ & 0.14 & 0.005 \\
\hline Neopham & $8 \pm 8$ & $13 \pm 10$ & $12 \pm 8$ & & \\
\hline \multicolumn{6}{|c|}{ Histidine (38-130 $\mu \mathrm{mol} /$ liter $)$} \\
\hline Aminosyn & $85 \pm 36$ & $92 \pm 14$ & $98 \pm 16$ & 0.85 & 0.77 \\
\hline Neopham & $66 \pm 14$ & $92 \pm 25$ & $103 \pm 21$ & & \\
\hline \multicolumn{6}{|c|}{ Taurine $(0-180 \mu \mathrm{mol} / \mathrm{liter})$} \\
\hline Aminosyn & $135 \pm 120$ & $119 \pm 78$ & $97 \pm 57$ & 0.81 & 0.63 \\
\hline Neopham & $174 \pm 174$ & $82 \pm 64$ & $79 \pm 60$ & & \\
\hline \multicolumn{6}{|c|}{ Tyrosine $(17-240 \mu \mathrm{mol} / \mathrm{liter})$} \\
\hline Aminosyn & $50 \pm 26$ & $25 \pm 9$ & $27 \pm 9$ & 0.48 & 0.08 \\
\hline \multirow{2}{*}{\multicolumn{6}{|c|}{ Aspartic acid $(0-32 \mu \mathrm{mol} /$ liter $)$}} \\
\hline & & & & & \\
\hline Áminosyn & $39 \pm 20$ & $45 \pm 13$ & $43 \pm 11$ & 0.10 & 0.01 \\
\hline Neopham & $31 \pm 15$ & $50 \pm 21$ & $58 \pm 20$ & & \\
\hline \multicolumn{6}{|c|}{ Glutamic acid $(0-320 \mu \mathrm{mol} / \mathrm{liter})$} \\
\hline Aminosyn & $190 \pm 128$ & $255 \pm 125$ & $282 \pm 98$ & 0.40 & 0.0008 \\
\hline Neopham & $249 \pm 171$ & $285 \pm 154$ & $324 \pm 169$ & & \\
\hline \multicolumn{6}{|c|}{ Glutamine $(430-1300 \mu \mathrm{mol} /$ liter $)$} \\
\hline Aminosyn & $255 \pm 169$ & $300 \pm 168$ & $304 \pm 138$ & 0.96 & 0.84 \\
\hline Neopham & $228 \pm 217$ & $287 \pm 201$ & $312 \pm 202$ & & \\
\hline \multicolumn{6}{|c|}{ Alanine (11-770 $\mu \mathrm{mol} / \mathrm{liter})$} \\
\hline Aminosyn & $214 \pm 83$ & $360 \pm 77$ & $376 \pm 81$ & 0.16 & 0.03 \\
\hline \multirow{2}{*}{\multicolumn{6}{|c|}{ Arginine $(40 \mu \mathrm{mol} /$ liter $)$}} \\
\hline & & & & & \\
\hline Aminosyn & $26 \pm 25$ & $96 \pm 84$ & $140 \pm 50$ & 0.24 & 0.02 \\
\hline Neopham & $39 \pm 38$ & $809 \pm 48$ & $104 \pm 45$ & & \\
\hline \multicolumn{6}{|c|}{ Glycine $(60-350 \mu \mathrm{mol} / \mathrm{liter})$} \\
\hline Aminosyn & $325 \pm 117$ & $675 \pm 196$ & $699 \pm 142$ & 0.0001 & 0.0001 \\
\hline Neopham & $299 \pm 145$ & $350 \pm 107$ & $416 \pm 109$ & & \\
\hline \multicolumn{6}{|c|}{ Proline $(110-430 \mu \mathrm{mol} /$ liter $)$} \\
\hline Aminosyn & $110 \pm 33$ & $272 \pm 93$ & $284 \pm 66$ & 0.34 & 0.13 \\
\hline Neopham & $132 \pm 55$ & $264 \pm 107$ & $328 \pm 144$ & & \\
\hline \multicolumn{6}{|c|}{ Serine $(43-230 \mu \mathrm{mol} /$ liter $)$} \\
\hline Aminosyn & $160 \pm 67$ & $248 \pm 72$ & $271 \pm 84$ & 0.62 & 0.45 \\
\hline Neopham & $151 \pm 72$ & $223 \pm 86$ & $354 \pm 74$ & & \\
\hline
\end{tabular}

${ }^{a}$ Represents normal values recalculated from Ghadimi and Pecora. ${ }^{17}$

TABLE X

Mean serum alkaline phosphatase, total and direct bilirubin, LDH, and SGOT in neonates treated uith Aminosyn and Neopham (n = 16)

\begin{tabular}{|c|c|c|c|c|c|}
\hline & \multirow{2}{*}{ Day 1} & \multirow{2}{*}{ Week 1} & \multirow{2}{*}{ Week 2} & \multicolumn{2}{|c|}{ p Values } \\
\hline & & & & Between group & Within group \\
\hline \multicolumn{6}{|c|}{ Alkaline phosphatase $(30-115 \mathrm{U} / \text { liter })^{a}$} \\
\hline Aminosyn & $134 \pm 36$ & $163 \pm 49$ & $252 \pm 36$ & 0.10 & 0.0001 \\
\hline Neopham & $135 \pm 46$ & $241 \pm 93$ & $349 \pm 119$ & & \\
\hline \multicolumn{6}{|c|}{ LDH (100-225 U/liter) } \\
\hline Aminosyn & $1531 \pm 1687$ & $380 \pm 315$ & $560 \pm 507$ & 0.25 & 0.007 \\
\hline Neopham & $992 \pm 462$ & $545 \pm 200$ & $364 \pm 98$ & & \\
\hline \multicolumn{6}{|c|}{ SGOT (8 40 U/liter) } \\
\hline Aminosyn & $510 \pm 1036$ & $35 \pm 18$ & $31 \pm 6$ & 0.27 & 0.15 \\
\hline Neopham & $147 \pm 113$ & $66 \pm 83$ & $39 \pm 11$ & & \\
\hline \multicolumn{6}{|c|}{ Total bilirubin $(0.3-1.2 \mathrm{mg} / \mathrm{dl})$} \\
\hline Aminosyn & $6.4 \pm 4.6$ & $5.5 \pm 4.2$ & $1.9 \pm 1.1$ & 0.48 & 0.0001 \\
\hline Neopham & $5.5 \pm 4.0$ & $10.0 \pm 6.1$ & $2.8 \pm 2.5$ & & \\
\hline \multicolumn{6}{|c|}{ Direct bilirubin $(0.0-0.3 \mathrm{mg} / \mathrm{dl})$} \\
\hline Aminosyn & $0.5 \pm 0.4$ & $0.4 \pm 0.3$ & $0.5 \pm 0.5$ & 0.50 & 0.81 \\
\hline Neopham & $0.3 \pm 0.3$ & $0.9 \pm 0.8$ & $0.8 \pm 0.8$ & & \\
\hline
\end{tabular}

- Values in parentheses represent normal values. 
Plasma Aminograms (Essential Amino Acids)

The plasma aminogram data for essential amino acids are presented in Tables VI and VII for the neonatal and total patient analyses, respectively. $P$-Values are listed for both between-group (Neopham is Aminosyn) and within-group (changes during the course of the study period) analyses.
Veonatal Analysis. Significant between-group differences were found for three amino acids, namely, isoleucine, methionine, and valine. Significant within group increases occurred with isoleucine, leucine, methionine, and valine in both the Aminosyn and Neopham patient groups. The levels of all the amino acids increased in both study groups (Table VI).

Total Patient Analysis. The levels of all the amino

TABLE XI

Mean serum alkaline phosphatase, total and direct bilirubin, $L D H$ and SGOT in patients treated with Aminosyn or Neopham (n $=24)$

\begin{tabular}{|c|c|c|c|c|c|}
\hline & \multirow{2}{*}{ Day 1} & \multirow{2}{*}{ Week 1} & \multirow{2}{*}{ Week 2} & \multicolumn{2}{|c|}{$p$ Values } \\
\hline & & & & Between group & Within group \\
\hline \multicolumn{6}{|c|}{ Alkaline phosphatase $(30-115 \mathrm{U} / \text { liter })^{a}$} \\
\hline Aminosyn & $126 \pm 34$ & $168 \pm 48$ & $259 \pm 31$ & 0.21 & 0.0001 \\
\hline Neopham & $786 \pm 557$ & $199 \pm 93$ & $283 \pm 126$ & & \\
\hline \multicolumn{6}{|c|}{ LDH (100-225 U/liter) } \\
\hline Aminosyn & $1244 \pm 1523$ & $468 \pm 397$ & $500 \pm 405$ & 0.16 & 0.006 \\
\hline Neopham & $786 \pm 557$ & $488 \pm 236$ & $360 \pm 133$ & & \\
\hline \multicolumn{6}{|c|}{ SGOT $(8-40 \mathrm{U} /$ liter $)$} \\
\hline Aminosyn & $393 \pm 902$ & $39 \pm 17$ & $31 \pm 5$ & 0.34 & 0.07 \\
\hline \multirow{2}{*}{\multicolumn{6}{|c|}{ Total bilirubin $(0.3-1.2 \mathrm{mg} / \mathrm{dl})$}} \\
\hline & & & & & \\
\hline Aminosyn & $5.8 \pm 4.5$ & $5.6 \pm 3.8$ & $4.1 \pm 2.2$ & 0.99 & 0.0001 \\
\hline Neopham & $3.6 \pm 4.0$ & $7.9 \pm 6.6$ & $2.0 \pm 2.3$ & & \\
\hline \multicolumn{6}{|c|}{ Direct bilirubin $(0.0-0.3 \mathrm{mg} / \mathrm{dl})$} \\
\hline Aminosyn & $0.4 \pm 0.4$ & $0.5 \pm 0.4$ & $1.2 \pm 0.8$ & 0.92 & 0.36 \\
\hline Neopham & $0.2 \pm 0.3$ & $0.7 \pm 0.8$ & $0.6 \pm 0.7$ & & \\
\hline
\end{tabular}

${ }^{a}$ Values in parentheses represent normal values.

TABLE XII

BUN, plasma ammonia, serum total protein, and albumin levels in neonates treated with Aminosyn or Neopham $(n=16)$

\begin{tabular}{|c|c|c|c|c|c|}
\hline & \multirow{2}{*}{ Day 1} & \multirow{2}{*}{ Week 1} & \multirow{2}{*}{ Week 2} & \multicolumn{2}{|c|}{$p$ Values } \\
\hline & & & & Between group & Within group \\
\hline \multicolumn{6}{|c|}{ BUN $(8-20 \mathrm{mg} / \mathrm{dl})^{a}$} \\
\hline Aminosyn & $8.5 \pm 3.7$ & $10.8 \pm 4.4$ & $11.3 \pm 2.1$ & 0.25 & 0.21 \\
\hline Neopham & $14.0 \pm 5.0$ & $12.2 \pm 4.9$ & $17.1 \pm 11.4$ & & \\
\hline \multicolumn{6}{|c|}{ Ammonia $(17-80 \mu \mathrm{g} / \mathrm{dl})$} \\
\hline Aminosyn & $154 \pm 186$ & $165 \pm 78$ & $91 \pm 44$ & 0.73 & 0.10 \\
\hline Neopham & $97 \pm 79$ & $180 \pm 151$ & $137 \pm 88$ & & \\
\hline \multicolumn{6}{|c|}{ Total protein $(6.0-8.0 \mathrm{~g} / \mathrm{dl})$} \\
\hline Aminosyn & $4.6 \pm 0.5$ & $5.0 \pm 0.7$ & $5.1 \pm 0.9$ & 0.71 & 0.001 \\
\hline Neopham & $4.2 \pm 0.9$ & $4.9 \pm 0.8$ & $5.0 \pm 0.6$ & & \\
\hline \multicolumn{6}{|c|}{ Albumin $(3.4-4.8 \mathrm{~g} / \mathrm{dl})$} \\
\hline Aminosyn & $3.9 \pm 0.4$ & $3.2 \pm 0.6$ & $3.2 \pm 0.7$ & 0.86 & 0.001 \\
\hline Neopham & $2.7 \pm 0.5$ & $3.2 \pm 0.5$ & $3.2 \pm 0.6$ & & \\
\hline
\end{tabular}

Values in parentheses represent normal values.

TABLE XIII

BUN, plasma ammonia, serum total protein, and albumin levels in patients treated with Aminosyn or Neopham $(n=24)$

\begin{tabular}{|c|c|c|c|c|c|}
\hline & \multirow{2}{*}{ Day 1} & \multirow{2}{*}{ Week 1} & \multirow{2}{*}{ Week 2} & \multicolumn{2}{|c|}{$p$ Values } \\
\hline & & & & Between group & Within group \\
\hline \multicolumn{6}{|c|}{ BUN $(8-20 \mathrm{mg} / \mathrm{dl})^{\circ}$} \\
\hline Aminosyn & $7.6 \pm 3.5$ & $12.4 \pm 5.5$ & $13.3 \pm 2.3$ & 0.94 & 0.23 \\
\hline Neopham & $11.4 \pm 5.4$ & $9.3 \pm 4.7$ & $12.6 \pm 9.9$ & & \\
\hline \multicolumn{6}{|c|}{ Ammonia $(17-80 \mu \mathrm{g} / \mathrm{dl})$} \\
\hline Aminosyn & $119 \pm 163$ & $215 \pm 171$ & $123 \pm 66$ & 0.09 & 0.01 \\
\hline Neopham & $71 \pm 72$ & $152 \pm 136$ & $120 \pm 87$ & & \\
\hline \multicolumn{6}{|c|}{ Total protein $(6.0-8.0 \mathrm{~g} / \mathrm{dl})$} \\
\hline Aminosyn & $4.7 \pm 0.5$ & $5.2 \pm 0.7$ & $5.1 \pm 0.7$ & 0.81 & 0.002 \\
\hline Neopham & $: \quad$ & $5.1 \pm 0.8$ & $5.3 \pm 0.8$ & & \\
\hline \multicolumn{6}{|c|}{ Albumin $(3.4-4.8 \mathrm{~g} / \mathrm{dl})$} \\
\hline Aminosyn & $3.0 \pm 0.5$ & $3.3 \pm 0.5$ & $3.2 \pm 0.5$ & 0.93 & 0.02 \\
\hline Neopham & $2.9 \pm 0.7$ & $3.1 \pm 0.5$ & $3.2 \pm 0.6$ & & \\
\hline
\end{tabular}

${ }^{a}$ Values in parentheses represent normal values. 
TABLE XIV

Mean serum sodium, chloride, potassium, calcium. and magnesium in neonates treated uith Aminosyn or Neopham ( $n=16$ )

\begin{tabular}{|c|c|c|c|c|c|}
\hline & \multirow{2}{*}{ Day 1} & \multirow{2}{*}{ Week 1} & \multirow{2}{*}{ Week 2} & \multicolumn{2}{|c|}{ p Values } \\
\hline & & & & Between group & Within group \\
\hline \multicolumn{6}{|c|}{ Sodium (137-149 mEq/liter $)^{a}$} \\
\hline Aminosyn & $139 \pm 4$ & $143 \pm 3(22)$ & $138 \pm 2$ & 0.08 & 0.03 \\
\hline Neopham & $136 \pm 7$ & $139 \pm 6(37)$ & $136 \pm 6$ & & \\
\hline \multicolumn{6}{|c|}{ Chloride (99-111, mEq/liter) } \\
\hline Aminosyn & $107 \pm 6$ & $110 \pm 2$ & $112 \pm 5$ & 0.11 & 0.10 \\
\hline Neopham & $105 \pm 5$ & $11-\ldots$ & $105 \pm 5$ & & \\
\hline \multicolumn{6}{|c|}{ Potassium (3.5-5.0 mEq/liter) } \\
\hline Aminosyn & $4.5 \pm 0.9$ & $5.1 \pm 11 . x$ & $5.6 \pm 0.1$ & 0.96 & 0.74 \\
\hline Neopham & $5.3 \pm 1.3$ & $4.9 \pm 1.1$ & $5.0 \pm 1.0$ & & \\
\hline \multicolumn{6}{|c|}{ Calcium $(8.5-10.5 \mathrm{mg} / \mathrm{dl})$} \\
\hline Aminosyn & $8.8 \pm 0.8$ & $9.9 \pm 1.2$ & $9.8 \pm 0.4$ & 0.40 & 0.0001 \\
\hline Neopham & $7.9 \pm 0.9$ & $9.2 \pm 1.0$ & $9.9 \pm 1.0$ & & \\
\hline \multicolumn{6}{|c|}{ Magnesium (1.5-2.3 mEq/liter) } \\
\hline Aminosyn & $1.53 \pm 0.25$ & $2.07 \pm 0.22$ & $1.80 \pm 0.0$ & 0.59 & 0.0001 \\
\hline Neopham & $1.46 \pm 0.25$ & $1.91 \pm 0.35$ & $2.12 \pm 0.39$ & & \\
\hline
\end{tabular}

"Values in parentheses represent normal values.

TABLE XV

Mean serum sodium, chloride, potassium, calcium, and magnesium levels in pati.

\begin{tabular}{|c|c|c|c|c|c|}
\hline & \multirow{2}{*}{ Day 1} & \multirow{2}{*}{ Week 1} & \multirow{2}{*}{ Week 2} & \multicolumn{2}{|c|}{$p$ Values } \\
\hline & & & & Between group & $\bar{W}_{i}$ \\
\hline \multicolumn{6}{|c|}{ Sodium $(137-149 \mathrm{mEq} / \text { liter })^{a}$} \\
\hline Aminosyn & $140 \pm 7$ & $140 \pm 6$ & $138 \pm 4$ & 0.08 & 0.25 \\
\hline Neopham & $136 \pm 6$ & $138 \pm 5$ & $136 \pm 5$ & & \\
\hline \multicolumn{6}{|c|}{ Chloride (99-111 mEq/liter) } \\
\hline Aminosyn & $106 \pm 7$ & $105 \pm 10$ & $103 \pm 10$ & 0.72 & 0.99 \\
\hline Neopham & $105 \pm 4$ & $106 \pm 6$ & $103 \pm 6$ & & \\
\hline \multicolumn{6}{|c|}{ Potassium $(3.5-5.0 \mathrm{mEq} /$ liter $)$} \\
\hline Aminosyn & $4.6 \pm 0.9$ & $5.2 \pm 1.0$ & $5.4 \pm 0.5$ & 0.36 & 0.92 \\
\hline Neopham & $5.0 \pm 1.2$ & $4.7 \pm 1.0$ & $4.6 \pm 1.0$ & & \\
\hline \multicolumn{6}{|c|}{ Calcium $(8.5-10.5 \mathrm{mg} / \mathrm{dl})$} \\
\hline Aminosyn & $8.8 \pm 0.9$ & $9.9 \pm 1.1$ & $9.8 \pm 0.4$ & 0.13 & 0.0001 \\
\hline Neopham & $8.2 \pm 0.8$ & $9.0 \pm 0.9$ & $9.5 \pm 1.0$ & & \\
\hline \multicolumn{6}{|c|}{ Magnesium (1.5-2.3 mEq/liter) } \\
\hline Aminosyn & $1.51 \pm 0.23$ & $2.07 \pm 0.20$ & $1.80 \pm 0.0$ & 0.75 & 0.0001 \\
\hline Neopham & $1.56 \pm 0.30$ & $1.96 \pm 0.33$ & $1.94 \pm 0.36$ & & \\
\hline
\end{tabular}

${ }^{a}$ Values in parentheses represent normal values.

acids increased in both study groups. Between- and within-group analyses were identical to those of the neonatal analysis for the essential amino acids (Table VII).

\section{Plasma Aminograms (Nonessential Amino Acids)}

The plasma aminogram analysis for the non-essential amino acids is presented to Table VIII (neonatal analy. sis) and Table IX (total patient analysis).

Neonatal Analysis. The only amino acid which was significantly different between the two study groups was glycine. Significant within-group changes vccurred with a number of amino acids. Significantly decreased levels of cystine occurred in the Aminosin-treated patients. while tyrosine decreased significantly in both the Aminosyn and Neopham groups. Significant increases oc curred in aspartic acid, glutamic acid, glutamine, arginine, glycine, and proline in both patient groups. Histidine, alanine, and serine increased during the course of therapy in both groups. but not signiticantly. Taurine levels decreased in both the Aminosyn and Neopham groups, but not significantly (Table VIII).

Total Patient Analysis. The only significant betweengroup difference was in glycine concent ration, which was significantly elevated in the Aminosyn-treated patients. Within-group significant increases occurred in cystine in the Neopham patients, while aspartic acid, glutamic acid, alanine, arginine, and glycine were significantly increased in both the Neopham and Aminosyn groups. In the Aminosyn group, cystine was significanti it.reased. Taurine and tyrosine decreased in both $g_{l}$ it not significantly: in contrast. histidine. glutamunt froline, and serine were increased insignificantly in both the Aminosyn and neopham groups (Table IX).

\section{Hematological Data}

Neonatal Analysis. There were no significant differences between treatment aroups for any of the hematological param.

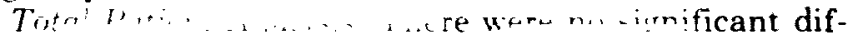
feren wentratment group- he hematologica parameters studied. 


\section{Biochemical Data}

Mean. standard deviation, and significance levels for between-and within-group analysis of biochemical data are presented in Tables $\mathrm{X}$ through XVII.

Neonatal Analysis. There were no significant betweengroup differences in alkaline phosphatase, LDH, SGOT. total and direct bilirubin. Within-group significant decreases were seen in alkaline phosphatase, LDH, and total bilirubin for both the Aminosyn and Neopham groups (Table $\mathrm{X}$ ).

There were no significant between-group differences in BUN, plasma ammonia, serum total protein, or albumin levels. Within-group analyses showed a significant increase in total protein and albumin in the Neopham treatment group (Table XII).

There were no significant between-group differences in serum sodium, chloride, potassium, calcium, or magnesium levels in either study group. Serum sodium, calcium, and magnesium increased significantly in both groups during the course of the study (Table XIV).

There were no significant between-group differences in serum glucose, triglyceride, cholesterol, or phosphorus levels; however, initial uric acid values were significantly elevated in the Neopham group. Within-group significant increases occurred in cholesterol levels, while uric acid levels significantly decreased in both treatment groups (Table XVI).

Total Patient Analysis. There were no significant between-group differences in alkaline phosphatase, $\mathrm{LDH}$, SGOT. and total and direct bilirubin. There were significant within-group decreases in LDH and total bilirubin in both treatment groups, and in alkaline phosphatase in the Neopham treatment group (Table XI).

There were no significant between-group differences in BUN, plasma ammonia, serum total protein, or albumin levels. Within-group analyses indicated a significant increase in ammonia, total protein, and albumin levels in the Neopham and Aminosyn treatment groups (Table XIII).

There were no significant between-group differences in serum sodium, chloride, potassium, calcium, or magnesium levels in either group. Calcium and magnesium increased significantly in both groups (Table XV).

There were no significant between group differences in serum glucose, triglyceride, cholesterol, phosphorus, or uric acid levels. Significant within-group increases

TABLE XVI

Mean serum glucose, triglycerides, cholesterol, phosphorus, and uric acid levels in neonates treated with Aminosyn or Neopham ( $n=16$ )

\begin{tabular}{|c|c|c|c|c|c|}
\hline & \multirow{2}{*}{ Day 1} & \multirow{2}{*}{ Week 1} & \multirow{2}{*}{ Week 2} & \multicolumn{2}{|c|}{$p$ Values } \\
\hline & & & & Between group & Within group \\
\hline \multicolumn{6}{|c|}{ Glucose $(73-115 \mathrm{mg} / \mathrm{dl})^{a}$} \\
\hline Aminosyn & $100 \pm 57$ & $94 \pm 22$ & $93 \pm 23$ & 0.98 & 0.53 \\
\hline Neopham & $84 \pm 44$ & $89 \pm 26$ & $102 \pm 29$ & & \\
\hline \multicolumn{6}{|c|}{ Triglycerides $(40-150 \mathrm{mg} / \mathrm{dl})$} \\
\hline Aminosyn & $98 \pm 18$ & $101 \pm 43$ & $82 \pm 62$ & 0.75 & 0.34 \\
\hline Neopham & $90 \pm 41$ & $112 \pm 50$ & $118 \pm 32$ & & \\
\hline \multicolumn{6}{|c|}{ Cholesterol $(0-250 \mathrm{mg} / \mathrm{dl})$} \\
\hline Aminosyn & $97 \pm 41$ & $185 \pm 24$ & $249 \pm 28$ & 0.93 & 0.0001 \\
\hline Neopham & $67 \pm 24$ & $208 \pm 67$ & $238 \pm 88$ & & \\
\hline \multicolumn{6}{|c|}{ Phosphorus $(2.4-4.5 \mathrm{mg} / \mathrm{dl})$} \\
\hline Aminosyn & $5.7 \pm 0.8$ & $6.3 \pm 2.0$ & $6.2 \pm 1.1$ & 0.43 & 0.86 \\
\hline Neopham & $5.8 \pm 1.0$ & $6.1 \pm 2.0$ & $5.7 \pm 1.5$ & & \\
\hline \multicolumn{6}{|c|}{ Uric acid $(3.9-9.0 \mathrm{mg} / \mathrm{dl})$} \\
\hline Aminosyn & $4.2 \pm 0.6$ & $3.5 \pm 0$ & $3.5 \pm 0.0$ & 0.04 & 0.0005 \\
\hline Neopham & $6.7 \pm 2.4$ & $3.1 \pm 0.6$ & $3.2 \pm 0.6$ & & \\
\hline
\end{tabular}

- Values in parentheses represent normal values.

TABLE XVII

Mean serum glucose, triglycerides, cholesterol, phosphorus, and uric acid levels in patients treated with Aminosyn or Neopham ( $n=24$ )

\begin{tabular}{|c|c|c|c|c|c|}
\hline & \multirow{2}{*}{ Day 1} & \multirow{2}{*}{ Week 1} & \multirow{2}{*}{ Week 2} & \multicolumn{2}{|c|}{$p$ Values } \\
\hline & & & & Between group & Within group \\
\hline \multicolumn{6}{|c|}{ Glucose $(73-115 \mathrm{mg} / \mathrm{dl})^{\circ}$} \\
\hline Aminosyn & $106 \pm 49$ & $89 \pm 22$ & $78 \pm 19$ & 0.42 & 0.88 \\
\hline Neopham & $95 \pm 41$ & $102 \pm 42$ & $107 \pm 29$ & & \\
\hline \multicolumn{6}{|c|}{ Triglycerides $(40-150 \mathrm{mg} / \mathrm{dl})$} \\
\hline Aminosyn & $95 \pm 34$ & $124 \pm \pi 1$ & $119 \pm 78$ & 0.98 & 0.04 \\
\hline Neopham & $96 \pm 48$ & $132 \pm 72$ & $129 \pm 61$ & & \\
\hline \multicolumn{6}{|c|}{ Cholesterol $(0-250 \mathrm{mg} / \mathrm{dl})$} \\
\hline Aminosyn & $98 \pm 40$ & $221 \pm 66$ & $284 \pm 74$ & 0.24 & 0.0001 \\
\hline Neopham & $79 \pm 33$ & $187 \pm 67$ & $217 \pm 83$ & & \\
\hline \multicolumn{6}{|c|}{ Phosphorus $(2.4-4.5 \mathrm{mg} / \mathrm{dl})$} \\
\hline Aminosyn & $5.3 \pm 1.1$ & $6.3 \pm 1.7$ & $6.1 \pm 1.0$ & 0.14 & 0.57 \\
\hline Neopham & $5.1 \pm 1.3$ & $5.2 \pm 2.1$ & $4.8 \pm 1.7$ & & \\
\hline \multicolumn{6}{|c|}{ Eric acid $(3.9-9.0 \mathrm{mg} / \mathrm{dl})$} \\
\hline Aminusyn & $3.8 \pm 0.9$ & $3.2 \pm 0.6$ & $3.5 \pm 0.0$ & 0.11 & 0.0003 \\
\hline Neopham & $5.7 \pm 2.5$ & $3.2 \pm 0.7$ & $3.0 \pm 0.4$ & & \\
\hline
\end{tabular}

\footnotetext{
- Values in parentheses represent normal values.
} 
occurred in cholesterol levels, whereas uric acid decreased significantly in both treatment groups (Table XVII).

\section{CONCLUSIONS}

Although nitrogen balances have to be viewed with caution in this study due to the difficulty with quantitative urine collections and the unaccounted losses of nitrogen through non-urinary routes, the similarity in nutrient intake, weight gain, and nitrogen retention in both groups indicates that Neopham and Aminosyn are equally effective in promoting growth in a TPN program. The weight increase per day in the Neopham neonatal group was lower $(0.32 \pm 3.79 \%$ compared to $0.87 \pm 2.33 \%$ in the Aminosyn group), but this change was not statistically significant. The nitrogen balance data was also slightly lower in the Neopham group $(285 \pm 135 \mathrm{mg} / \mathrm{kg} /$ day, $70.4 \%$ retention, compared with the Aminosyn group, $340 \pm 71.8 \mathrm{mg} / \mathrm{kg} /$ day, $7.18 \%$ retention).

The plasma aminogram analyses reflected, in general, the composition of the respective parent solutions. All of the essential amino acids (isoleucine, leucine, lysine, methionine, phenylalanine, threonine, and valine) increased over the time course of the study in both treatment groups. Significant increases in isoleucine, methionine, and valine in the Aminosyn vs the Neopham treatment group probably reflect the higher concentration of these amino acids in the Aminosyn preparation. Four of the essential amino acids (isoleucine, leucine, methionine, and valine) increased significantly in both treatment groups over the course of the study.

Analysis of the nonessential amino acids revealed a significantly greater increase in glycine in the Aminosyn group, probably because of the higher concentration of this amino acid in Aminosyn. Significant changes occurred in cystine, tyrosine, aspartic acid, glutamic acid, glutamine, arginine, glycine, and proline. Decreased plasma levels of cystine in the Aminosyn patients probably reflect the lack of cysteine in the Aminosyn solution. Levels of taurine decreased over the course of the study; neither amino acid solution contains taurine. Neopham contains $410 \mathrm{mg} / \mathrm{dl}$ of aspartic acid and $710 \mathrm{mg} / \mathrm{dl}$ of glutamic acid, whereas Aminosyn contains neither. However, the two patient groups had similar plasma levels of these amino acids.

Therefore, it appears that the serum amino acid profile of these patients closely reflects the amino acid composition of the parent solution.

Hemoglobin, hematocrit, and the erythrocyte count all significantly decreased during the course of the study. Also, white blood cell count, especially neutrophils and immature bands, decreased. In contrast, the lymphocyte and platelet counts increased significantly. There is no obvious explanation for these changes; however, frequent blood sampling may explain the decreases in hemoglobin, hematocrit, and erythrocyte count.
The increase in alkaline phosphatase, total protein, and albumin were significant but not readily explainable, although all of these changes could result from the TPN therapy.

On the basis of this limited study, it appears that Neopham is as effective as Aminosyn in a TPN regimen in terms of weight gain, nitrogen balance, and overall clinical well-being. No serious side effects were seen with either amino acid solution. In addition, plasma aminograms appear to be a direct reflection of the amino acid composition of infused solution.

\section{ACKNOWLEDGMENT}

This study was supported by KabiVitrum, Inc., Alameda, California.

\section{REFERENCES}

1. Borrensen HC, Coran AG, Knutrud O: Metabolic results of parenteral feeding in neonatal surgery: $A$ balanced parenteral feeding program based on a synthetic L-amino acid solution and a commercial fat emulsion. Ann Surg 172:291-301, 1970

2. Coran AG, Weintraub WH: Peripheral intravenous feeding without fat in neonatal surgery. J Pediatr Surg 12:195-199, 1977

3. Coran AG: The long-term intravenous feeding of infants using peripheral veins. J Pediatr Surg 8:801-807, 1977

4. Coran AG: Total intravenous feeding of infants and children without the use of a central venous catheter. Ann Surg 179:445-449, 1974

5. Connors RH, Coran AG, Wesley JR: Studies on the efficacy and toxicity of a new fat emulsion in pediatric parenteral nutrition. JPEN 4:384-386, 1980

6. Coats DA, Waynard AT: Long-term parenteral nutrition. IN Parenteral Nutrition, Meng HC, Law KH (eds). Charles C Thomas, Springfield, IL, 1970

7. Jurgens P, Bansi HW, Dolif D, et al: Experimental results of clinical evaluations of amino acid solutions in parenteral nutrition. IN Parenteral Nutrition, Meng HC, Law KH (eds). Charles C Thomas, Springfield, IL, 1970

8. Jurgens P, Dolif D: Experimental results of parenteral nutrition with amino acids. IN Parenteral Nutrition, AW Wilkinson (ed). Churchill-Livingstone, Edinburgh-London, 1972

9. Heird WC, Winters RW: Total parenteral nutrition. J Pediatr $86: 2-16,1975$

10. Klein RE, Habicht JP, Yarbrough C: Effects of protein-calorie malnutrition on mental development. IN Advances in Pediatrics, Schulman I (ed). Year Book Medical Publishers, Chicago, IL, 1971

11. Seward JF, Serdula MK: Task force on infant-feeding practices: Infant feeding and infant growth. Pediatrics 74:728-762, 1984

12. Stoch MB, Smythe PM: Does undernutrition during infancy inhibit brain growth and subsequent intellectual development? Arch Dis Child 38:546-552, 1963

13. Winick M: Malnutrition and brain development. J Pediatr 74:667679,1969

14. Martin CL: Growth and development of children maintained on total parenteral nutrition in the neonatal period. JAMA 29:481483,1974

15. Rosso P, Winick M: Malnutrition and growth: Relation of nutrition to physical and mental development. Pediatr Ann 2:33-43, 1973

16. Winick M: Nutrition and mental development. Med Clin N Am 54:1413-1429, 1970

17. Ghadimi H, Pecora P: Plasma amino acids after birth. Pediatrics 34:182-191, 1964 